\title{
INCIDENCIA DISTRIBUTIVA DEL SISTEMA TRIBUTARIO ARGENTINO
}

\author{
Fernández Felices, Diego* \\ Guardarucci, Isidro \\ Puig, Jorge
}

enviado: julio 2016 - aceptado: octubre 2016

\begin{abstract}
Resumen
El trabajo estudia la incidencia distributiva del sistema tributario argentino (por impuesto y a nivel agregado) haciendo uso de las dos últimas Encuestas Nacionales de Gasto de los Hogares correspondientes a los años 2004/05 y 2012/13. Al utilizar el ingreso per cápita familiar como indicador de bienestar, se obtiene que el sistema tributario ejerce mayor presión en ambas colas de la distribución, siendo constante en los deciles intermedios. Por otro lado, si se utiliza el consumo per cápita familiar como indicador, la incidencia resulta proporcional con excepción del último decil, en donde aumenta considerablemente. De este modo se pone de manifiesto la relevancia del indicador de bienestar elegido a la hora de realizar un análisis de incidencia tributaria.
\end{abstract}

Código JEL: H11, H22

Palabras clave: impuestos, incidencia distributiva, encuestas de gastos.

\begin{abstract}
This paper studies the distributive impact of the tax system in Argentina using the last two available Household Expenditure Surveys for 2004/05 and 2012/13. Calculations were made using both household per capita income and household per capita consumption as welfare indicators. By using household per capita income, the tax system puts relatively more pressure on both tails of the distribution, being

${ }^{*}$ UNLP, Facultad de Ciencias Económicas

Correo electrónico: diegoferfel@gmail.com, isidroguar@gmail.com, jorgeppuig@gmail.com
\end{abstract}


approximately constant for the intermediate deciles. On the other hand, choosing per capita consumption as welfare indicator makes the tax system roughly proportional, with the exception of the last decile, where tax pressure becomes much higher. These differences on the results make clear the relevance of the chosen welfare indicator for tax incidence analysis.

JEL Code: H11, H22.

Key words: taxes, tax incidence, expenditure surveys.

\section{INTRODUCCIÓN}

El papel central que juegan los impuestos en cuestiones relacionadas con la distribución del ingreso hace que los estudios sobre la incidencia del sistema impositivo sean de crucial importancia en cualquier debate sobre políticas públicas.

El presente trabajo realiza un análisis de incidencia distributiva del sistema tributario argentino haciendo uso de las dos últimas Encuestas Nacionales de Gasto de los Hogares (ENGHo) correspondientes a los años 2004/05 y 2012/13, publicadas por el Instituto Nacional de Estadísticas y Censo (INDEC). Se adopta el enfoque denominado de "usos y fuentes" mediante el cual la incidencia económica de los impuestos es calculada de acuerdo a un conjunto de supuestos ad hoc acerca de la distribución de las cargas tributarias de los distintos impuestos.

Los resultados encontrados en este trabajo ponen de manifiesto la importancia de la elección del indicador de bienestar considerado. Al utilizar el ordenamiento de los individuos por su nivel de ingresos, el sistema tributario ejerce una mayor presión en ambas colas de la distribución, siendo constante en los deciles intermedios. Por otro lado, al ordenar a los individuos por consumo, la presión tributaria resulta constante con excepción del último decil, en donde aumenta considerablemente.

La estructura del trabajo es la siguiente. La sección I describe la metodología tradicional de los estudios de incidencia distributiva y los datos utilizados. La sección II realiza una breve descripción de los impuestos que componen el sistema tributario argentino. La sección III constituye el núcleo del trabajo y presenta el análisis de incidencia de cada uno de los impuestos estudiados utilizando la ENGHo 2004/05. La sección IV replica los resultados utilizando la última ENGHo disponible, correspondiente a 2012/13. La sección V presenta las conclusiones. 


\section{METODOLOGÍA Y DATOS}

En todo estudio de incidencia distributiva es necesario comenzar precisando la unidad de análisis, y al respecto existen dos opciones igualmente válidas: el individuo y el hogar (Gasparini, 1998). Elegida la unidad de análisis, debe definirse el indicador de bienestar utilizado para ordenarla. Este aspecto se vuelve relevante dado que los análisis de incidencia suelen hacerse en términos del cálculo de la presión tributaria, entendida como el cociente entre el pago del impuesto soportado por el individuo (u hogar) y el indicador de bienestar elegido por el analista. La gran mayoría de los estudios de incidencia ordenan a los individuos a partir del ingreso corriente (anual o mensual, dependiendo de la fuente de información) agrupándolos en estratos. Sin embargo, de acuerdo con la visión intertemporal del consumo, los individuos tienen la posibilidad de trasladar consumo entre períodos, por lo cual su bienestar estará más relacionado con alguna medida de ingreso permanente que con el ingreso corriente. Es así que algunos estudios incluyen, como parte del análisis de incidencia, el uso del ingreso permanente a través de alguna variable proxy (en general el consumo corriente) como criterio para ordenar a la población, por considerarlo un mejor indicador del bienestar de los individuos (Gasparini, 1998; Santiere, Gómez Sabaini, y Rossignolo, 2002; Gaggero y Rossignolo, 2011) ${ }^{1}$.

Utilizar el ordenamiento por ingreso corriente puede sesgar los resultados por dos vías diferentes. Una de ellas surge al considerar como pobres a individuos que se encuentran en un período del ciclo de vida durante el cual sus ingresos son bajos. De acuerdo con la teoría del ciclo de vida, el flujo de ingresos de un individuo a lo largo de su vida activa presenta la forma de una $U$ invertida. El ingreso es bajo al inicio de la vida, incrementándose hasta alcanzar su máximo valor a mediados de la vida activa, para luego decrecer a medida que se acerca la edad de retiro. Un análisis de incidencia que no tome en cuenta la edad de los individuos daría por resultado una fuerte desigualdad entre ellos, aun cuando se tuviera la misma corriente de ingresos a lo largo de la vida. Por otro lado, el sesgo de los resultados puede deberse a que el ingreso de los individuos simplemente ha sufrido un shock transitorio negativo. Para resolver ambos problemas, los analistas aconsejan ordenar a los individuos según su ingreso permanente, lo cual puede conducir a resultados diferentes de los que se obtienen cuando el criterio para ordenar a la población es el ingreso corriente.

1 Ver Fullerton y Metcalf (2002) para una completa reseña de los diferentes enfoques de estudio vinculados a incidencia tributaria, incluida una discusión sobre el uso de indicadores de bienestar para el cálculo de incidencia tributaria en estudios empíricos. 
Una vez elegido el indicador de bienestar para ordenar a los individuos, el análisis de incidencia impositiva exige identificar a los agentes económicos que soportan la carga del impuesto (o grupo de impuestos) bajo análisis. Dado que la conducta económica de los agentes económicos puede verse alterada ante cambios en las condiciones de mercado provocados por los impuestos, la incidencia económica no necesariamente coincidirá con el responsable legal del impuesto.

Considerando todo lo anterior, en el presente trabajo se opta, como en la mayoría de los estudios de incidencia, por el individuo como unidad de análisis. Respecto del indicador de bienestar, se trabaja tanto con el ingreso corriente como con el consumo corriente ${ }^{2},{ }^{3}$. Para el cálculo de la incidencia distributiva propiamente dicho, se adoptó el enfoque de equilibrio parcial debido a sus menores requerimientos de información (en comparación con el enfoque de equilibrio general ${ }^{4}$ ) y que suele ser el más comúnmente adoptado por los estudios existentes en la literatura. De acuerdo a éste, la incidencia económica de los impuestos es calculada en base a un conjunto de supuestos ad hoc acerca de la distribución de las cargas tributarias de los distintos impuestos, ya sea sobre los usos o sobre las fuentes de ingreso. Dichos supuestos se realizan teniendo en cuenta principalmente la evidencia empírica disponible acerca de las elasticidades de oferta y demanda de los bienes y factores gravados, las cuales determinarán la facilidad o dificultad de aumentar los precios de los bienes que se comercializan, o de reducir la remuneración de los factores que se contratan. El presente trabajo hace uso de este enfoque y los supuestos de traslación adoptados coinciden en su mayoría con aquellos utilizados en estudios previos.

Finalmente para llevar a cabo las estimaciones se utilizó primariamente la Encuesta Nacional de Gastos de los Hogares de los años 2004/05 (ENGHo

2 Para aproximar el consumo se utilizó la variable de gasto registrada en la Encuesta Nacional de Gasto de los Hogares. En el caso de la medición del consumo corriente, resulta dificultoso obtener información del consumo de bienes durables, por lo cual no serán incluidos en este trabajo. Bajo el supuesto razonable de que el consumo de estos bienes se incrementa con el nivel de ingreso del individuo, su omisión en el cálculo del consumo estará subestimando el nivel de bienestar de los individuos de más altos ingresos y sesgando los resultados hacia una mayor progresividad.

3 Una cuestión relevante, asociada al uso de datos de ingresos y consumos declarados en encuestas, tiene que ver con la subdeclaración de los mismos. Si bien existen diferentes métodos para ajustar los datos ante la existencia de este fenómeno, la postura adoptada en este trabajo es la de utilizar los datos de la ENGHO sin realizar ajustes por subdeclaración. Para una discusión sobre distintos métodos de ajuste por subdeclaración y una justificación de la postura adoptada aquí, ver Gasparini, Cicowiez y Sosa Escudero (2013).

4 Una referencia clásica sobre la metodología de los modelos de equilibrio general computados es Shoven y Whalley (1992). 
2004/05), publicada por Instituto Nacional de Estadísticas y Censo (INDEC). Esta encuesta cuenta con la ventaja de proveer información sobre el gasto de los hogares, lo cual permite aproximar el consumo corriente de los hogares. A su vez se utiliza la ENGHo perteneciente a los años 2012/13 para un análisis de robustez temporal de la incidencia tributaria.

\section{EL SISTEMA TRIBUTARIO ARGENTINO5}

El gobierno nacional recauda más del $80 \%$ del total de los ingresos fiscales de la Nación, fundamentalmente a través de gravámenes sobre el consumo, los ingresos y el patrimonio. Dentro del primer rubro se encuentra el principal impuesto del sistema tributario argentino, el Impuesto al Valor Agregado (IVA), del cual proviene aproximadamente un cuarto de la recaudación total. Adicionalmente existen impuestos internos selectivos que gravan el consumo de bienes y servicios como combustibles, cigarrillos y bebidas, los cuales aportan alrededor de 5 puntos de recaudación.

Los impuestos a los ingresos consisten principalmente en el gravamen de las ganancias de personas físicas y el correspondiente a corporaciones o personas jurídicas. A su vez, el trabajo se grava a través de los aportes y contribuciones a la seguridad social. El primer gravamen aporta un $20 \%$ de la recaudación, mientras que el segundo aporta casi el $22 \%$.

El impuesto patrimonial de mayor relevancia lo constituye el impuesto a los créditos y débitos en cuenta corriente, que aporta el 5\% de la recaudación total.

Por último, el Gobierno Nacional recauda un $8 \%$ del total a través de impuestos sobre el comercio exterior mediante derechos de exportación e importación.

En lo que respecta al primer nivel subnacional de gobierno, se aprecia que el principal impuesto provincial en términos de recaudación es el impuesto sobre los ingresos brutos, que grava en virtud de la capacidad económica demostrada por el ejercicio de cierto tipo de oficio, actividad o profesión. Adicionalmente los gobiernos provinciales gravan los inmuebles, los automotores y las transacciones onerosas, estas últimas a través del impuesto a los sellos.

5 Véase http://www.mecon.gov.ar/sip/basehome/rectrib.htm. 


\section{RESULTADOS DE INCIDENCIA DISTRIBUTIVA DE LOS IMPUESTOS}

En esta sección se analiza la incidencia impositiva de los impuestos que conforman el sistema tributario argentino. En cada caso se especifica el supuesto de traslación utilizado y se presenta el cómputo de la incidencia utilizando el ingreso per cápita familiar y el consumo per cápita familiar como medidas alternativas de bienestar.

\section{III.1. Impuestos Nacionales \\ III.1.1. Impuesto al Valor Agregado (IVA)}

El IVA es un impuesto al consumo con una alícuota general (21\%), algunos bienes con tratamiento especial (ej. $10.5 \%$ sobre algunos alimentos y $27 \%$ sobre algunos servicios) y otros bienes exentos ${ }^{6}$. En consecuencia, el supuesto de traslación utilizado en este trabajo sostiene que la totalidad de la carga impositiva es soportada por los consumidores. De este modo, a efectos de computar la incidencia impositiva del IVA, se asignó a cada familia el pago total del mismo de acuerdo al consumo de bienes reflejado en la ENGHo, considerando las actividades alcanzadas, alícuotas y exenciones vigentes en la Ley 20.631, que establece su reglamentación.

La presión tributaria se calcula como el cociente entre la participación de cada decil en la recaudación del impuesto y la correspondiente participación de dicho decil en la distribución del bienestar. La Figura 1 muestra los valores de la presión tributaria calculados utilizando tanto el consumo como el ingreso como indicador de bienestar. Como se observa, los resultados en base al ingreso per cápita familiar (línea continua) muestran una caída sostenida de la presión tributaria a mayores ingresos, levemente reforzada en los deciles extremos, lo cual indica la regresividad del IVA al utilizar este indicador del bienestar. Esto puede explicarse por la baja capacidad de ahorro de las familias más pobres y la relativamente más alta capacidad de ahorro de las familias más ricas, en sentido corriente.

Los efectos recién comentados se ven alterados al utilizar como indicador de bienestar alguna variable que represente el ingreso permanente de las familias,

6 Al gravar el valor agregado en cada etapa de producción de bienes y servicios, su efecto equivale al de un impuesto a las ventas finales sobre todos los bienes, siempre que la alícuota impositiva en cada etapa del IVA sea uniforme y no existan bienes exentos. 
como el consumo per cápita familiar. De este modo, la regresividad del IVA se ve fuertemente atenuada, como lo muestra la línea punteada de la Figura $1^{7}$. Así, de la Figura 1 se desprende que el IVA resulta prácticamente proporcional utilizando el consumo per cápita como indicador de bienestar ${ }^{8}$.

Figura 1. Presión tributaria del Impuesto al Valor Agregado.

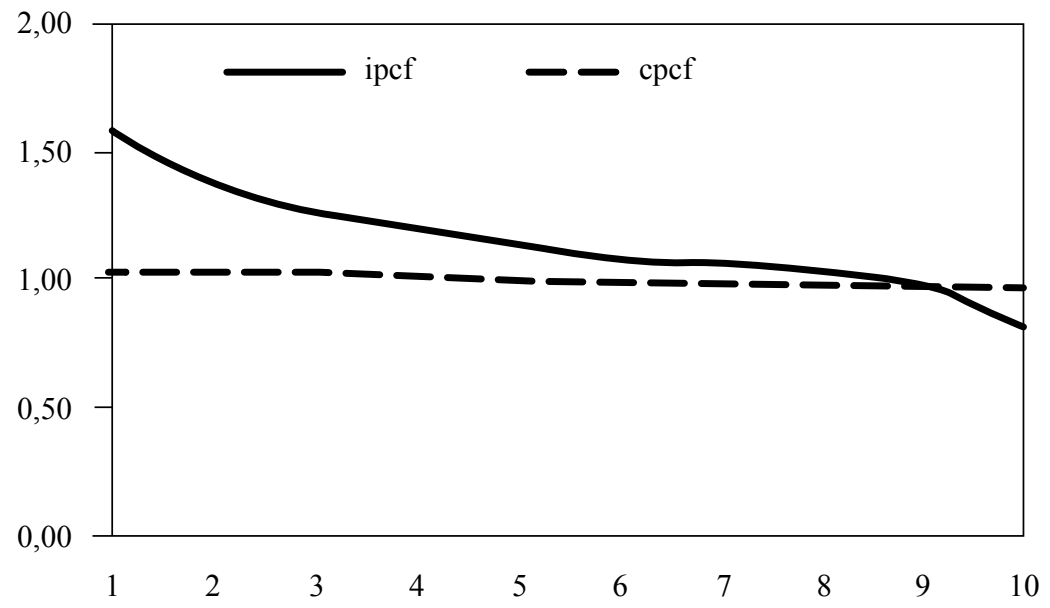

Fuente: elaboración propia en base a ENGHo 2004/05.

\section{III.1.2. Impuesto a las ganancias de personas físicas}

Este tributo grava los ingresos de las personas sin distinguir entre sus diferentes fuentes. Su capacidad de traslación depende principalmente de las elasticidades de la oferta de trabajo y del ahorro. El supuesto de traslación comúnmente realizado en la práctica, y seguido también en el presente trabajo, implica imputar la carga de este impuesto al responsable legal del mismo. A partir de los ingresos

7 Nótese que, por su estructura, el IVA debería resultar estrictamente proporcional computado de este modo, de no existir bienes exentos ni alícuotas diferenciales. Es por eso que todo apartamiento respecto de la proporcionalidad del IVA al utilizar el consumo per cápita familiar como indicador de bienestar, obedecerá necesariamente al patrón de consumo de bienes exentos del impuesto, o con alícuotas reducidas.

8 Este resultado está en línea con el obtenido por Gasparini (1998) utilizando diversas fuentes de datos, entre ellas la ENGHO de 1996. Dicha encuesta, a diferencia de las encuestas utilizadas aquí, no cuenta con datos desagregados a nivel de hogares o individuos. 
y de características laborales y familiares declarados en la ENGHo, se calculó el monto pagado por cada individuo de acuerdo a las escalas, deducciones y exenciones que establece la Ley $26.628^{9}$.

De la Figura 2 se desprende que este impuesto es altamente progresivo para ambas variables elegidas como indicadores de bienestar. El decil más rico absorbe más del $90 \%$ de la recaudación total del impuesto (99\% en el caso del ordenamiento de acuerdo al ingreso per cápita familiar). La presión tributaria resulta prácticamente nula para toda la distribución, con excepción del último decil. La fuerte progresividad del impuesto se ve levemente atenuada para el caso del consumo per cápita familiar.

Figura 2. Presión tributaria del Impuesto a las ganancias de personas físicas.

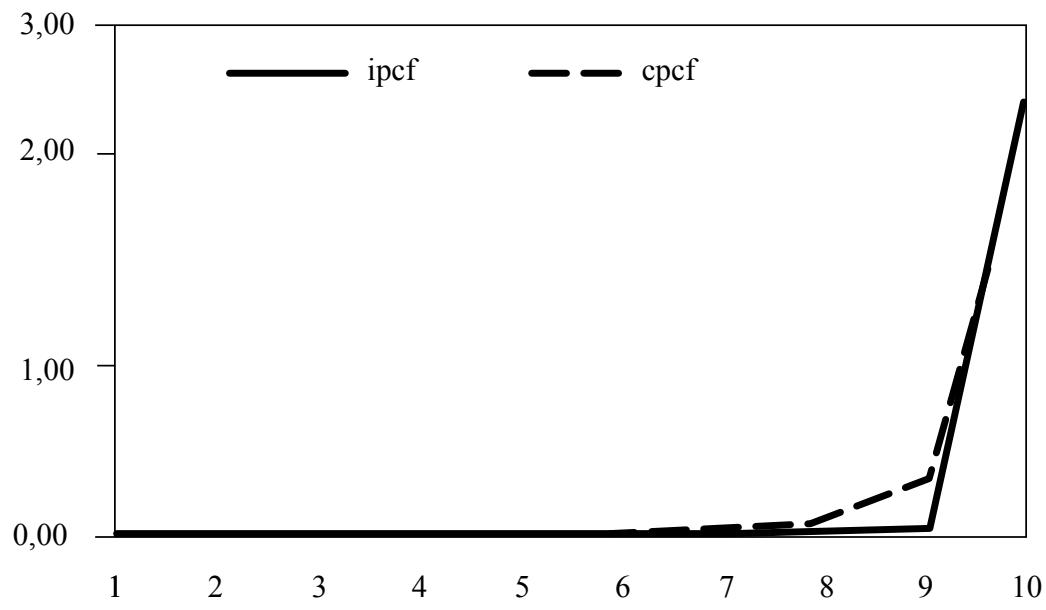

Fuente: elaboración propia en base a ENGHo 2004/05.

III.1.3. Impuesto a las ganancias de las empresas

La incidencia del impuesto a las ganancias de las empresas resulta mucho más controversial que la de ganancias a las personas físicas presentada en el apartado anterior. El peso de este tributo puede recaer sobre el capital en forma de una menor tasa de retorno después del impuesto, o puede ser trasladado en parte

9 Para una clara descripción de la Ley del Impuesto a las Ganancias, ver Díaz Bazán (2011). 
o totalmente al consumo de bienes producidos por las empresas gravadas, o a los factores complementarios al capital en la producción, típicamente al trabajo.

La posibilidad del capital de escapar al impuesto depende de su elasticidad de oferta ${ }^{10}$ y demanda. Si existen alternativas sustitutas a invertir en las empresas gravadas, el capital puede evitar el impuesto con relativa facilidad. Dado que el capital no es completamente móvil, se asume aquí que el mismo absorbe una parte de la carga del impuesto. Adicionalmente y bajo supuestos usuales, el trabajo soporta parte de la carga del impuesto, así como también puede trasladarse parte del impuesto hacia el consumo de los bienes producidos en los sectores gravados, al menos si se trata de bienes no transables.

Dada esta situación, se asume aquí que sólo 1/5 del impuesto es absorbido por el capital, mientras que los $4 / 5$ restantes recaen sobre el trabajo y el consumo en partes iguales ${ }^{11}$.

La distribución del capital en los sectores gravados se estimó a partir de la ENGHo donde se incluyen los ingresos de capital ${ }^{12},{ }^{13}$. La distribución del trabajo se realiza en base a los mismos procedimientos utilizados para evaluar la incidencia del impuesto al trabajo (detallados en el próximo apartado) mientras que la distribución del consumo total se realiza en base al gasto total de los hogares.

En la Figura 3 se aprecia que, considerando el ordenamiento por ingreso per cápita familiar, el impuesto a las ganancias de las empresas resulta progresivo, mientras que bajo un ordenamiento por consumo per cápita familiar resulta proporcional con la excepción del último decil.

10 Uno de los principales determinantes de la elasticidad de la oferta de este factor es su movilidad internacional.

11 Gasparini (1998) y Fernández Felices et al. (2014a) plantean otras alternativas sobre la combinación de estas proporciones. Aun así el impuesto a las ganancias de las empresas resulta progresivo para todas alternativas planteadas y el grado de progresividad es naturalmente mayor cuanto menor sea la traslación al consumo en relación al trabajo y, fundamentalmente, al capital

12 Contabilizar los ingresos de capital distinguiendo por categoría ocupacional (asalariado, cuentapropista y patrón o empleador) puede presentar inconvenientes debido a que, si bien la encuesta pide a cada individuo que clasifique sus ingresos entre salarios y honorarios provenientes de rentas, es probable que éstos confundan la respuesta. La subdeclaración representa un problema adicional para el cálculo de esta variable.

13 La variable de la ENGHO (irentas) capta "intereses, alquileres, rentas y dividendos" de manera conjunta sin discriminar entre las distintas fuentes. 
Figura 3. Presión tributaria del impuesto a las ganancias de las empresas.

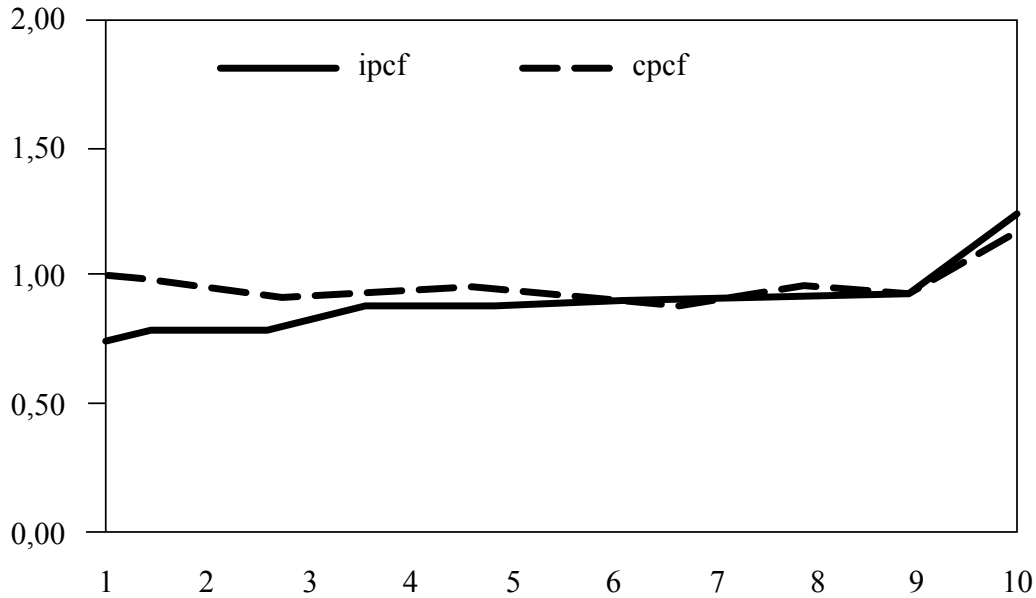

Fuente: elaboración propia en base a ENGHo 2004/05.

\section{III.1.4. Impuesto al trabajo}

Los impuestos al uso del factor trabajo son utilizados para cubrir buena parte del gasto en el sistema de seguridad social, el cual es financiado en una proporción menor por rentas generales ${ }^{14}$. El supuesto usual en buena parte de los estudios de incidencia impositiva consiste en imputar toda la carga de este impuesto a los trabajadores, más allá de que legalmente una parte del mismo recaiga sobre los empleadores en términos de cargas patronales. La justificación de este supuesto de traslación total reside básicamente en la relativamente baja elasticidad de la oferta de trabajo que surge de la evidencia empírica.

Para el cálculo de la incidencia distributiva de este impuesto se asignó a cada familia la carga imputada sobre los ingresos laborales declarados en la ENGHo. La Figura 4 muestra la proporcionalidad del impuesto al trabajo tomando el ingreso corriente como indicador de bienestar (ipcf) y la mayor regresividad del mismo al considerar el consumo como indicador (cpcf).

14 De acuerdo a la legislación, este impuesto se denomina Aportes y Contribuciones a la Seguridad Social. 
En el primer caso, la presión impositiva casi uniforme por deciles obedece a que el impuesto al trabajo opera prácticamente como un impuesto proporcional sobre los ingresos, siendo el ingreso por salarios el componente principal de los mismos para la mayoría de la población. Se observa una menor presión en los extremos de la distribución, lo cual puede obedecer a la participación de otras fuentes de ingreso distintas del salario para los individuos afectados (tales como transferencias, rentas e ingresos de jubilados o patrones). En el caso del ordenamiento por consumo per cápita familiar, la presión tributaria es notoriamente decreciente con el bienestar de la población, transformando al impuesto al trabajo en un impuesto claramente regresivo.

Figura 4. Presión tributaria del impuesto al trabajo.

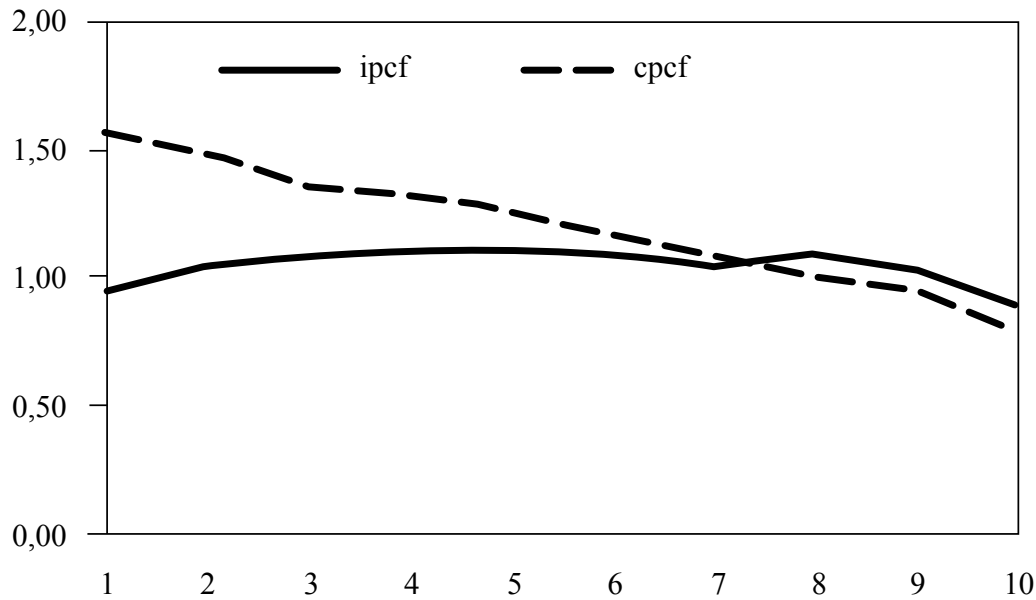

Fuente: elaboración propia en base a ENGHo 2004/05.

\section{III.1.5. Impuestos internos}

Estos impuestos gravan el consumo de ciertos bienes y servicios, como combustibles, cigarrillos, bebidas alcohólicas, ciertas bebidas sin alcohol, telefonía y seguros. Por lo tanto, el supuesto de incidencia general coincide con el realizado para el caso del IVA, asignando toda la carga tributaria a los consumidores de los bienes gravados. Los cómputos fueron realizados en base a la declaración de consumo de bienes de la ENGHo, las alícuotas y bases imponibles fijados por la Ley de Impuestos Internos $\mathrm{N}^{\circ} 24.674$. 
En la Figura 5 se puede apreciar que los impuestos internos, dado que en su mayoría recaen sobre el consumidor, son regresivos cuando se utiliza el ingreso corriente como indicador de bienestar, mientras que resultan proporcionales al utilizarse el consumo corriente ${ }^{15}$.

Figura 5. Presión tributaria de los impuestos internos.

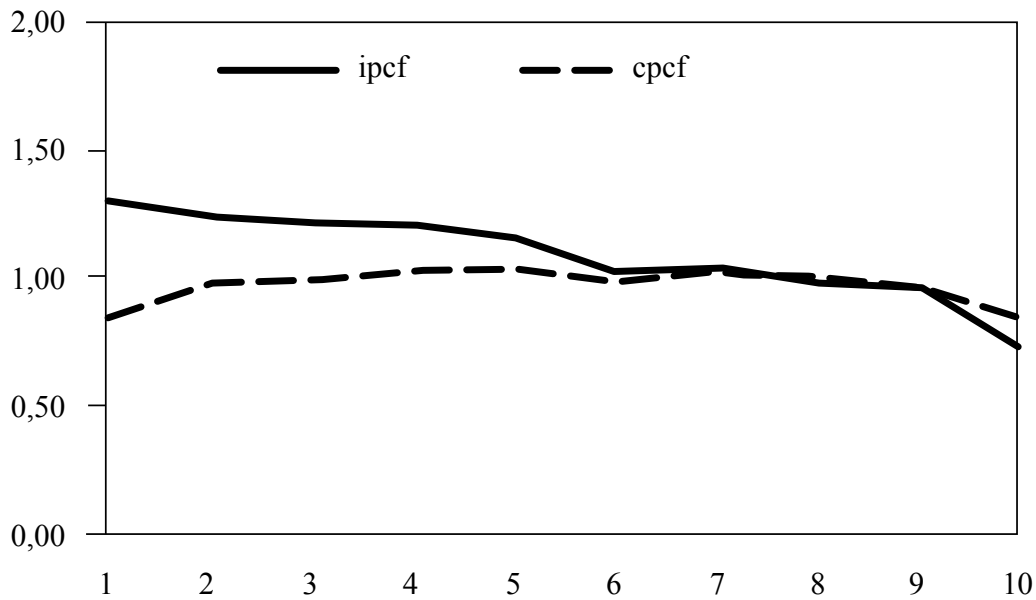

Fuente: elaboración propia en base a ENGHo 2004/05.

III.1.6. Derechos de exportación e importación ${ }^{16}$

Para analizar la incidencia de los derechos de exportación, se sigue el supuesto de que las mismas son soportadas en su totalidad por los productores agropecuarios. Para ello se utilizó la distribución funcional del ingreso de jefes de hogar cuya ocupación principal es el sector agropecuario, identificados a través del código de ocupación principal según el Clasificador Nacional de Ocupaciones (CNO) 2001 que provee la ENGHo.

Como se presenta en la Figura 6, los derechos de exportación son progresivos. Dicha progresividad es más acentuada cuando se toma el consumo per

15 Para una descripción detallada de cada uno de los impuestos internos por separado, véase Fernández Felices et al (2014a)

16 Se aclara aquí que en este trabajo se analizan únicamente los derechos de exportación sobre el sector agropecuario. 
cápita como indicador de bienestar (si bien en el último tramo de la distribución la presión es más alta si se ordena por ingreso).

Figura 6. Presión tributaria de los derechos de exportación.

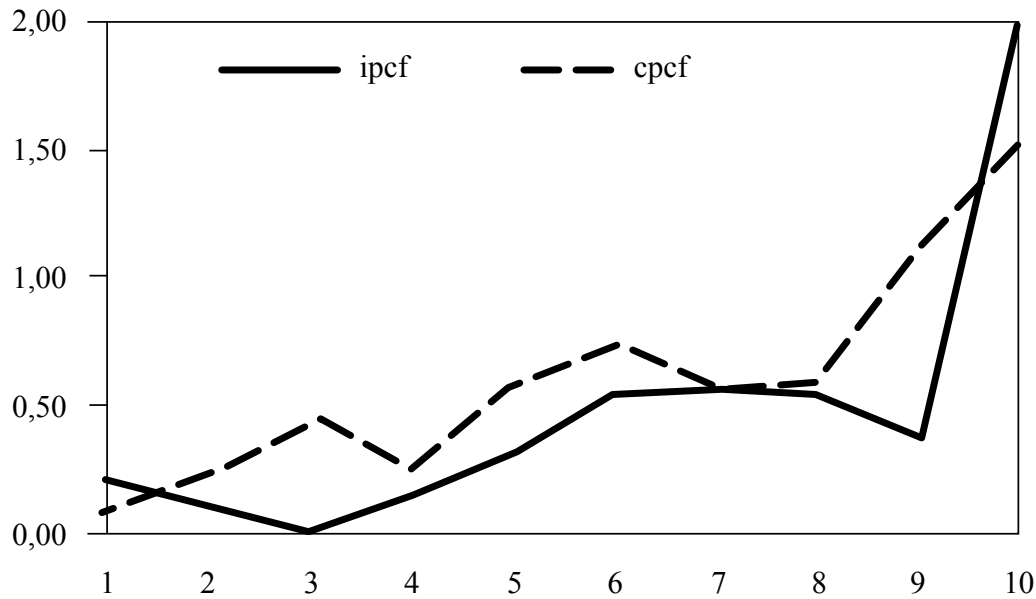

Fuente: elaboración propia en base a ENGHo 2004/05.

Figura 7. Presión tributaria de los derechos de importación.

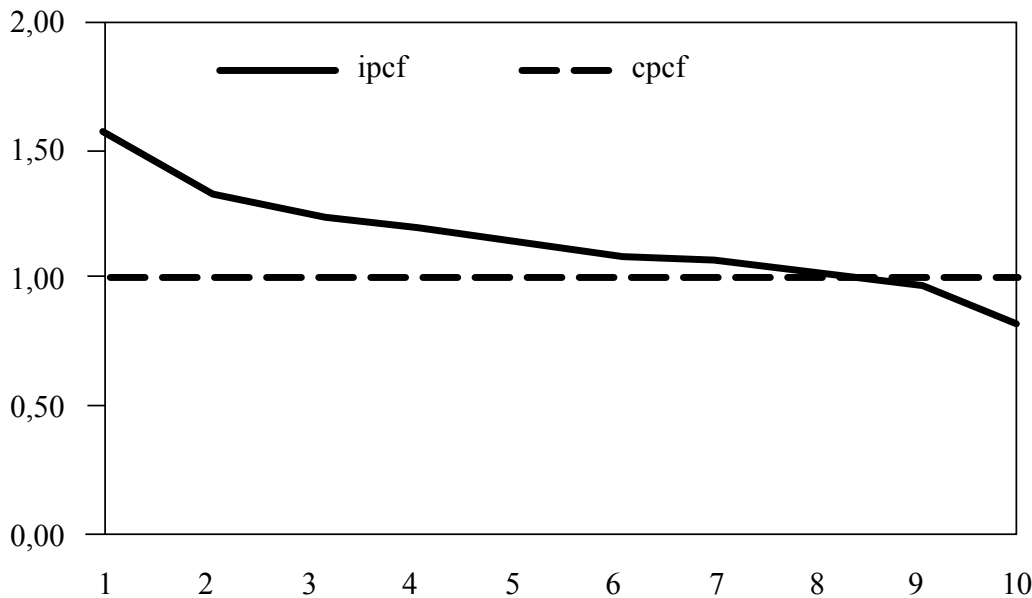

Fuente: elaboración propia en base a ENGHo 2004/05. 
Los aranceles sobre los productos importados recaen sobre aquellos agentes que consumen bienes gravados importados, ya sea directamente o a través del consumo de bienes y servicios que usan insumos importados gravados. Así, en este trabajo se asigna la carga de los derechos de importación de acuerdo a la distribución del consumo total. De este modo, los derechos de importación resultan estrictamente proporcionales si se ordena a los individuos por consumo, mientras que se tornan regresivos al ordenar por ingreso. Este resultado se puede observar en la Figura 7.

\section{III.1.7. Impuesto a los créditos y débitos en cuenta corriente}

Este impuesto grava los débitos y créditos en cuenta corriente bancaria y estableciendo que el tributo recaerá sobre los titulares de las cuentas respectivas, actuando las entidades financieras como agentes de liquidación y percepción. Debido a que es un tributo cuya carga en gran medida se traslada, se supone aquí que el mismo incide sobre el consumo global en un $80 \%$, mientras que el restante $20 \%$ lo hace sobre el ingreso global (este supuesto sigue al realizado en Gaggero y Rossignolo, 2011). Los resultados, presentados en la Figura 8 , indican que este tributo resulta marcadamente regresivo cuando se utiliza el ingreso como indicador de bienestar, tendiendo a la proporcionalidad cuando se ordena por consumo per cápita familiar.

Figura 8. Presión tributaria del impuesto a los créditos y débitos en cuenta corriente

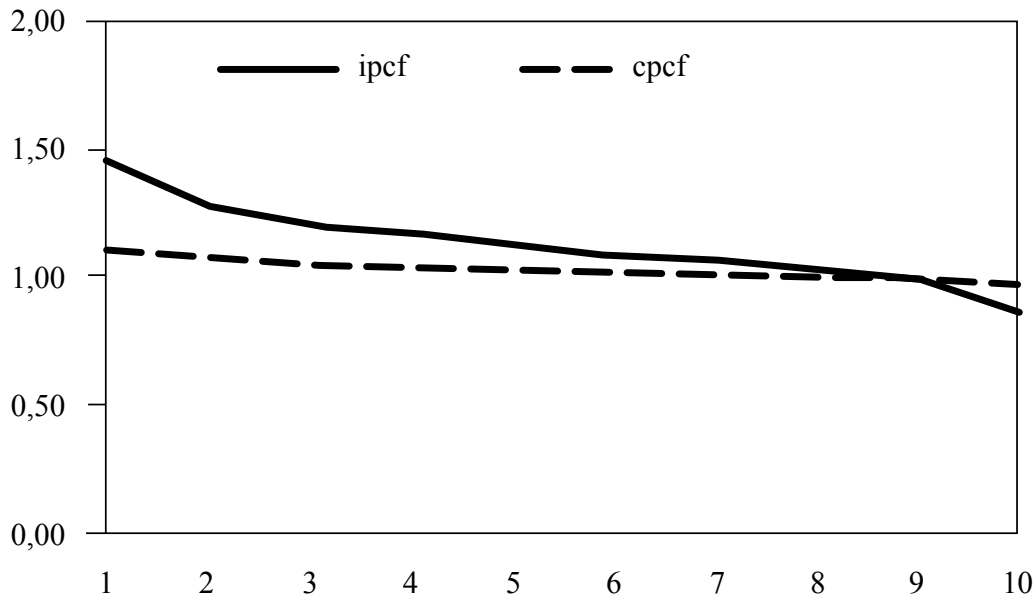

Fuente: elaboración propia en base a ENGHo 2004/05. 


\section{III.2. Impuestos Provinciales}

III.2.1. Impuesto sobre los ingresos brutos

El impuesto sobre los ingresos brutos representa el tributo provincial de mayor importancia en términos de recaudación. Es un impuesto que grava por etapas, recayendo en todas las etapas de producción y distribución de bienes y servicios. Por tal motivo, se lo suele clasificar dentro de la categoría de impuestos multifásicos acumulativos.

A la hora de analizar su incidencia y establecer algún supuesto sobre la traslación del gravamen, es sumamente relevante tener en cuenta que, en el caso de los bienes transables, el tributo recaerá en buena parte sobre los factores productivos, dado que no se puede trasladar por completo precio final del bien. Distinto es el caso de un sector no transable, donde se admite una mayor transmisión a los precios.

Para el cálculo propiamente dicho, se contó con información de recaudación para la provincia de Buenos Aires por rama de actividad. Para el resto de las jurisdicciones, la misma fue aproximada a partir de datos de valor de la producción y de las alícuotas vigentes en los distintos sectores (Mongan, Pollera y Salim, 2008). De acuerdo a lo ya mencionado, se asume que en los sectores productores de bienes transables la incidencia del impuesto recae 2/3 sobre los asalariados y $1 / 3$ sobre los empleadores, mientras que en los sectores productores de servicios no transables la incidencia recae íntegramente sobre los consumidores.

Dados estos supuestos, los resultados observados en la Figura 9 señalan que el impuesto a los ingresos brutos es regresivo si se tiene en cuenta un ordenamiento por ingreso per cápita familiar y se vuelve proporcional a la hora de ordenar por consumo per cápita familiar. 
Figura 9. Presión tributaria del impuesto a los ingresos brutos.

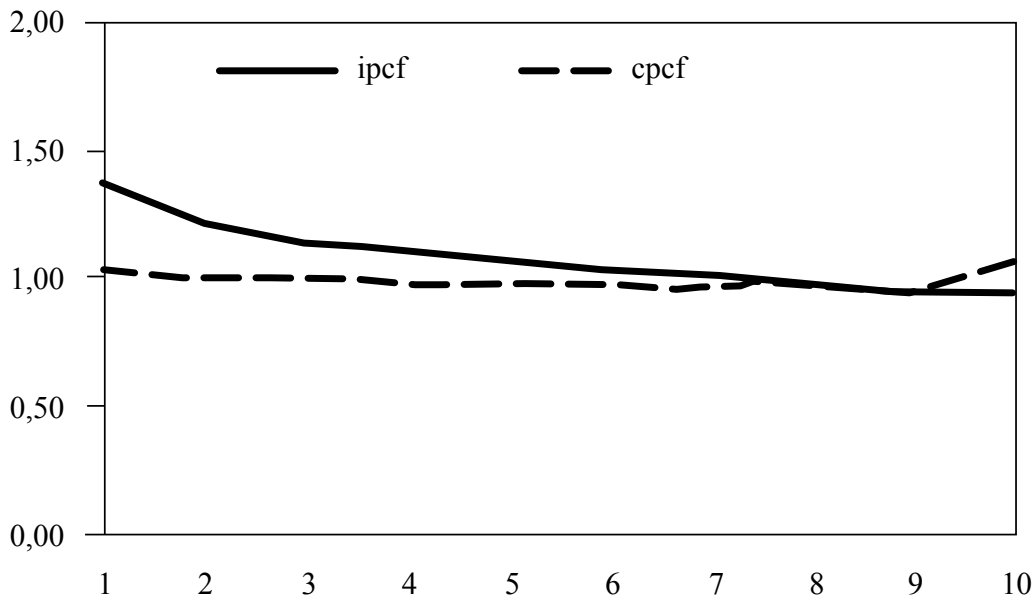

Fuente: elaboración propia en base a ENGHo 2004/05.

\section{III.2.2. Impuesto inmobiliario}

El impuesto inmobiliario es otro de los tributos que recaudan las provincias argentinas, gravando tanto las propiedades urbanas como las rurales.

A la hora de analizar la incidencia del impuesto inmobiliario urbano, la literatura plantea dos alternativas. Por un lado se sostiene que los que soportan el impuesto son los propietarios o inquilinos de los inmuebles, ya que este impuesto afecta la demanda de propiedades dado que la oferta es inelástica al menos en el mediano plazo. Por otra parte existe una postura que sostiene que el impuesto opera con una lógica similar al impuesto al capital (Gasparini, 1998). Respecto del inmobiliario rural, se coincide en que el mismo recae sobre el propietario de la tierra.

Planteado lo anterior, en lo que refiere al cálculo de incidencia, en este trabajo se utilizará un criterio basado en estimaciones ya existentes, donde se asume que el $64,5 \%$ recae sobre la vivienda, el $18,7 \%$ se asume como comercial urbano y se asigna según el consumo global, mientras que el 16,6\% restante se asumió como correspondiente al sector rural y se asignó de acuerdo a la distribución del ingreso de patrones jefes de hogar de la ENGHo (este criterio se adopta siguiendo a Gaggero y Rossignolo, 2011). La Figura 10 presenta los resultados de incidencia 
para el impuesto inmobiliario. Es posible apreciar que el mismo resulta progresivo bajo ambos indicadores de bienestar.

Figura 10. Presión tributaria del impuesto inmobiliario.

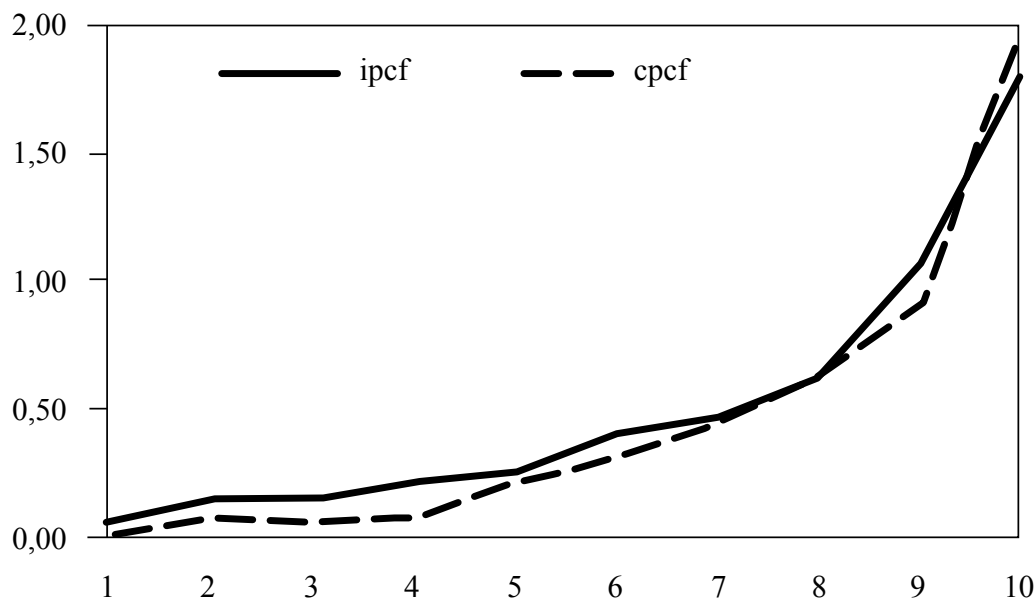

Fuente: elaboración propia en base a ENGHo 2004/05.

Figura 11. Presión tributaria del impuesto automotor.

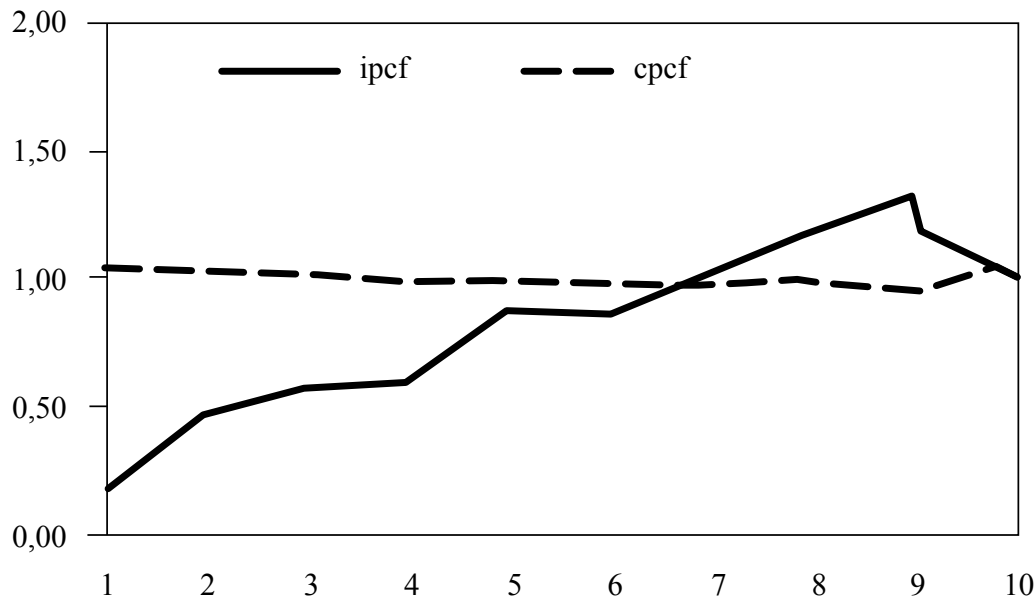

Fuente: elaboración propia en base a ENGHo 2004/05. 


\section{III.2.3. Impuesto automotor}

En el caso del impuesto a los automotores, se supone que el mismo recae sobre los propietarios de vehículos radicados en cada una de las provincias. Para estimar su incidencia, se utilizó la distribución de pagos del seguro automotor disponible en la ENGHo. Como puede observarse en la Figura 11, el impuesto resulta claramente progresivo a la hora de ordenar por ingreso per cápita familiar, mientras que se vuelve proporcional al ordenar por consumo per cápita.

\section{III.2.4. Impuesto a los sellos}

El último de los impuestos provinciales analizados aquí es el impuesto a los sellos, que se recauda a través del cobro de actos, contratos y operaciones de carácter oneroso. Algunos estudios sostienen que el impuesto es regresivo dado que, con una lógica similar a la del impuesto sobre los ingresos brutos, distribuyen la recaudación por consumo o producción (Santiere, Gomez Sabaini y Rossignolo, 2002). Por otra parte, otros estudios sostienen que los actos gravados, principalmente sobre transacciones de bienes registrables y contratos, distan mucho de asimilarse a las alcanzadas con los impuestos generales a los consumos o la producción. Así, asignando por rubros relacionados con dichas transacciones registrables y contratos, el impuesto resulta progresivo (Velasco y Tobes, 2012).

Figura 12. Presión tributaria del impuesto a los sellos.

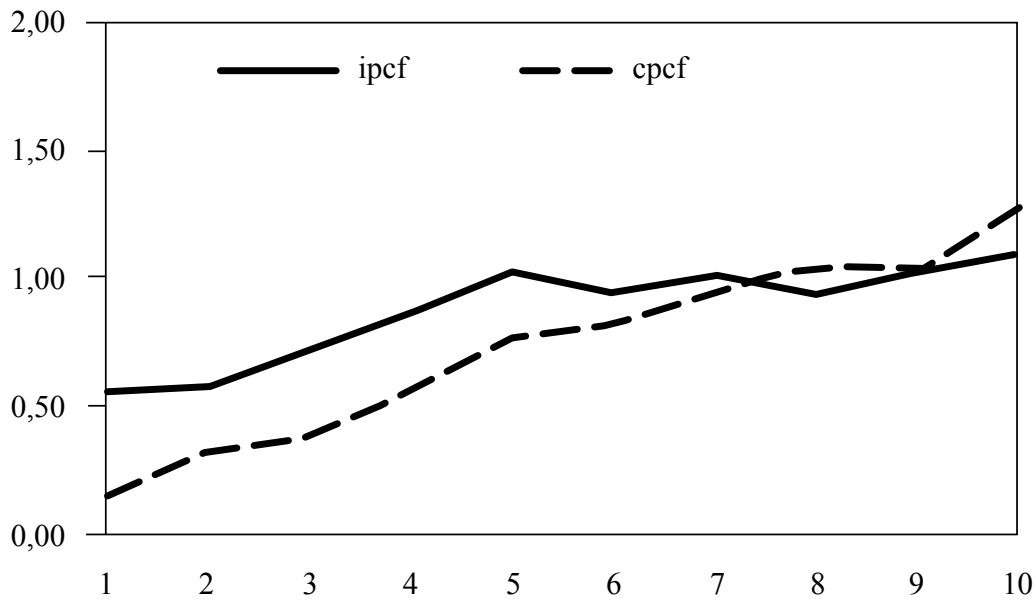

Fuente: elaboración propia en base a ENGHo 2004/05. 
En este trabajo, aprovechado el detalle con el que cuenta la ENGHo, se sigue la segunda de las líneas antes descriptas y se distribuye la carga del impuesto según el gasto en compra-venta de bienes registrables, gastos por obtención y reproducción de documentos, alquileres y cargos por tarjetas de crédito, entre otras. Los resultados presentados en la Figura 12 muestran al impuesto a los sellos como progresivo bajo ambos tipos de ordenamiento (ingreso corriente o consumo), siendo más pronunciado este efecto si se ordena por consumo per cápita familiar.

\section{INCIDENCIA GLOBAL DEL SISTEMA TRIBUTARIO ARGENTINO}

Con el objeto de evaluar el efecto que genera el conjunto de los impuestos del sistema tributario argentino sobre la distribución del bienestar, se presenta aquí el cálculo de la carga tributaria por decil de la totalidad de los impuestos considerados en las secciones anteriores.

Figura 13. Presión tributaria global del sistema tributario argentino.

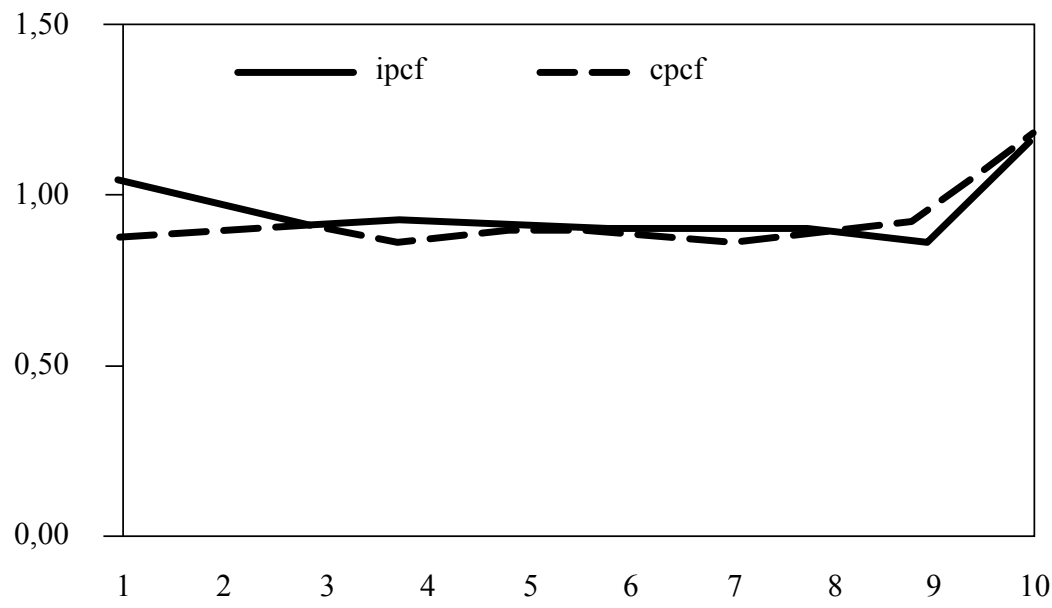

Fuente: elaboración propia en base a ENGHo 2004/05.

En la Figura 13 se observa que, al ordenar a los individuos por consumo, el sistema tributario argentino resulta proporcional con una ligera progresividad en el último decil. Al ordenar a los individuos por su nivel de ingreso, se observa una leve regresividad para la cola inferior de la distribución, mientras que nuevamente la presión sobre el $10 \%$ de mayores ingresos se acentúa. Estos resultados pueden racionalizarse 
dada la elevada participación de los impuestos sobre el consumo, el considerable peso relativo de la imposición sobre los ingresos y la aplicación de los derechos de exportación, estos últimos concentrados en el segmento de mayor poder adquisitivo.

\section{COMPARACION DE LA INCIDENCIA EN BASE A LA ULTIMA ENGHo 2012/13}

Esta sección presenta de manera sintética los resultados detalladamente expuestos anteriormente pero surgidos de la utilización de la última ENGHo para los años 2012/13. Esto permite comparar los resultados obtenidos ante cambios en la encuesta. Con el fin de sintetizar los resultados, se utiliza únicamente el ingreso per cápita familiar como indicador de bienestar y se exponen los resultados obtenidos a través del índice de Kakwani ${ }^{17}$.

Se puede observar que el IVA resulta regresivo y que dicha regresividad ha aumentado entre 2004/05 y 2012/13 (Figura 14). El resultado opuesto se observa para el impuesto a las ganancias de las personas físicas, mostrando un aumento en su progresividad. Los impuestos internos resultan regresivos al igual que los derechos de importación, mientras que los derechos de exportación son progresivos.

A nivel provincial se observan cambios considerables en la incidencia distributiva de los impuestos al comparar entre encuestas, ya que el valor estimado del índice de Kakwani se revierte tanto para el impuesto a los sellos como para el impuesto automotor ${ }^{18}$. El impuesto a los ingresos brutos es regresivo mientras que el impuesto inmobiliario se muestra marcadamente progresivo.

Finalmente, al estimar la incidencia global del sistema tributario se observa que el mismo es levemente progresivo, resultado que se explica por el fuerte efecto del impuesto a las ganancias y de los derechos de exportación.

17 El índice de Kakwani (K) se define como K = Gt - Ga, siendo Gt el coeficiente de Gini correspondiente a la curva de concentración del impuesto en consideración (o conjunto de impuestos) y Ga el coeficiente de Gini estándar correspondiente a la distribución del ingreso pre-impuestos. Así, un índice $\mathrm{K}$ negativo (positivo) indica un impuesto regresivo (progresivo).

18 Fernández Felices et al. (2014b) presentan las distribuciones de las cargas impositivas de ambos impuestos. Se puede apreciar allí una disminución de la incidencia relativa sobre los deciles más altos entre 2004/05 y 2012/13, lo que sugiere la existencia de cambios en los patrones de gastos en los rubros utilizados para calcular la incidencia de cada tributo. 
Figura 14. Incidencia distributiva del sistema tributario argentino. Años 2004/05 versus 2012/13. Índice de Kakwani.

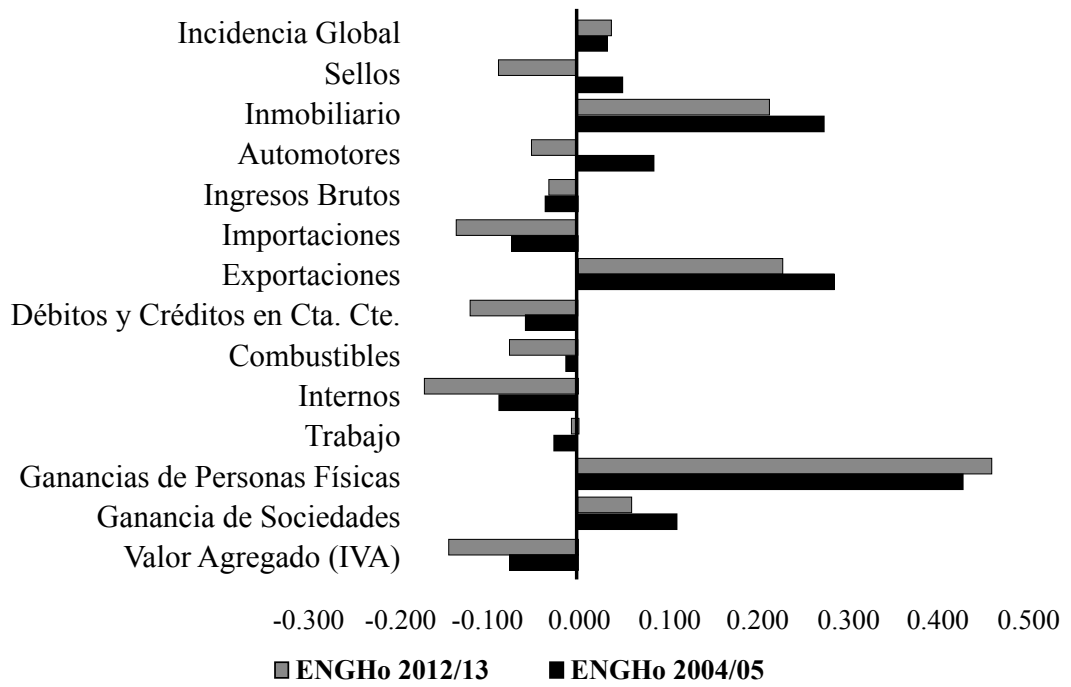

Fuente: elaboración propia en base a ENGHo 2004/05 y ENGHo 2012/13.

\section{CONCLUSIONES}

El presente trabajo analiza la incidencia distributiva del sistema tributario argentino, utilizando las dos últimas Encuestas de Gastos de los Hogares (ENGHo) correspondientes a los años 2004/05 y 2012/13. Adoptando un enfoque de equilibrio parcial y supuestos estándar de traslación para los diferentes impuestos del sistema tributario, puede observarse que en buena parte de los impuestos analizados existen marcadas diferencias en los cálculos de incidencia según cual sea la variable elegida como indicador de bienestar (ingreso per cápita familiar o consumo per cápita familiar). Al analizar el sistema tributario agregado, se encuentra que el mismo resulta aproximadamente proporcional para los deciles intermedios utilizando ambas variables, con una mayor regresividad observada en el primer decil al utilizar ingreso per cápita familiar. Ambas variables permiten observar una mayor presión tributaria para el decil más alto de la población.

Los impuestos en los que el supuesto de traslación utilizado implica asignar 
la carga tributaria al consumo del bien gravado (IVA, Ingresos Brutos, internos, derechos de importación, débitos bancarios) resultan en general regresivos al utilizar ingreso per cápita familiar como medida de bienestar. Sin embargo, se advierte una marcada tendencia hacia la proporcionalidad de los mismos al utilizar el consumo per cápita familiar como medida de bienestar.

El impuesto a las ganancias de las personas físicas resulta fuertemente progresivo bajo ambas medidas utilizadas como indicador de bienestar. Los impuestos al trabajo resultan proporcionales al utilizar ingreso per cápita como medida de bienestar y regresivos al utilizar consumo per cápita.

Con excepción de los impuestos a los sellos y a automotores, los resultados cualitativos en términos de progresividad o regresividad de los diferentes impuestos no se ven modificados al comparar las dos encuestas de gastos analizadas en este trabajo (2004/05 y 2012/13).

\section{REFERENCIAS BIBLIOGRAFICAS}

Diaz Bazán, T, (2011). Medidas de desigualdad a partir de datos impositivos. (Tesis de Maestría en Economía sin publicar). Recuperado de http://www. depeco.econo.unlp.edu.ar/maestria/tesis/084-tesis-diaz-bazan.pdf

Fernández Felices, D. Guardarucci, I. \& Puig, J. (2014a). Incidencia distributiva del sistema tributario argentino. Departamento de Economía, Facultad de Ciencias Económicas, Nacional de La Plata. Documento de Trabajo No. 105. Recuperado de http://www.depeco.econo.unlp.edu.ar/doctrab/ doc105.pdf

Fernández Felices, D. Guardarucci, I. \& Puig, J., (2014b). Impuestos y distribución del ingreso en Argentina: análisis y propuestas de reforma para el sistema tributario. Departamento de Economía, Facultad de Ciencias Económicas, Universidad Nacional de La Plata (mimeo).

Fullerton, D., \& Metcalf, G., Tax Incidence. (2002). En Handbook of Public Economics (vol. 4, pp 1787-1872). Amsterdam: Elsevier.

Gaggero, J. \& Rossignolo, D. (2011). Impacto del presupuesto sobre la equidad. Cuadro de Situación (Argentina, 2010). Centro de Economía y Finanzas para el Desarrollo de la Argentina.

(CEFID-AR). Documento de Trabajo No. 40.

Gasparini, L. (1998). Incidencia distributiva del sistema impositivo argentino. En La reforma tributaria en la Argentina. Tomo II (pp. 367-416). Fundación de Investigaciones Económicas Latinoamericanas. 
Gasparini, L., Cicowiez, M. \& Sosa Escudero, W. (2013). Pobreza y Desigualdad en América Latina. Conceptos, herramientas y aplicaciones. Buenos Aires: Temas Grupo Editorial.

Gómez Sabaini, J.C., Santieri, J.J. \& Rossignolo, D. (2002). La equidad distributiva y el sistema tributario: un análisis para el caso argentino. Serie Gestión Pública No. 20. Santiago de Chile: ILPES-CEPAL.

Mongan, J. C. Salim, L. \& Pollera, M. (2008). Hacia un indicador del incumplimiento del impuesto sobre los ingresos brutos en la provincia de Buenos Aires. Trabajo publicado en los Anales de las $41^{\circ}$ Jornadas Internacionales de Finanzas Públicas [CD]. Córdoba, Argentina: Facultad de Ciencias Económicas, Universidad Nacional de Córdoba.

Shoven, J., \& Whalley, J., (1992). Applying General Equilibrium, Cambridge: Cambridge University Press.

Velasco, P. \& Tobes, M., (2012). Incidencia del impuesto a los sellos en la provincia de Buenos Aires. Trabajo publicado en los Anales de las $45^{\circ}$ Jornadas Internacionales de Finanzas Públicas [CD]. Córdoba, Argentina: Facultad de Ciencias Económicas, Universidad Nacional de Córdoba.

(C) 2016 por los autores; licencia otorgada a la Revista Estudios Económicos. Este artículo es de acceso abierto y distribuido bajo los términos y condiciones de una licencia Atribución-No Comercial 3.0 Unported (CC BY-NC 3.0) de Creative Commons. Para ver una copia de esta licencia, visite http://creativecommons.org/ licenses/by-nc/3.0/ 